\section{Introduction to Nanotechnology}

Charles P. Poole Jr. and Frank J. Owens

(John Wiley \& Sons Inc.,

Hoboken, N.J., 2003)

402 pages; $\$ 84.95$

ISBN 0-471-07935-9

Poole and Owens intend this book to be an overview of nanotechnology to provide researchers with exposure to areas outside their immediate expertise or for a broad introduction for technical managers and administrators. The presentation is well geared to their target audiences. The selection and breadth of topics is appropriate, as few researchers are likely to be an expert in the wide variety of topics presented. Seldom will a researcher be a specialist in both fullerenes and ferromagnets, so this introduction to a variety of nanotopics is timely and at an appropriate level.

The introductory chapter provides a historical overview and context for nanotechnology developments. Chapters 2 and 3 are brief introductions to solid-state physics and to characterization techniques that make the study and development of nanoscale molecules and systems possible.

In the introduction to solid-state physics in Chapter 2, the authors deviate from the traditional approach to fcc structures and focus the discussion on the smallest theoretical nanoparticles and other larger nanoparticle configurations leading to structural magic numbers. The authors introduce energy bandgaps and phonons early, which is excellent. The treatment is by no way deep, but provides introduction and definition to concepts important in solid-state physics. Appropriate attention is paid to semiconductors, as these systems are foundational for many nanoscale devices.

The third chapter presents a number of the technological advances that allow the pursuit of nanotechnology. While the instrumentation and techniques for developing nanostructures is only mentioned briefly, appropriate detail is provided regarding experimental techniques for their study and characterization. There is an introduction to x-ray diffraction, mass spectrometry, Raman spectroscopy, and MRI, with some references to more in-depth treatments.

Chapters 4 through 12 then present a variety of nanoscale structures and phenomena, including nanoparticles, carbon nanostructures (including clusters and nanotubes), ferromagnetism, confined semiconductor structures (e.g., quantum wells, wires, and dots), polymers, selfassembled materials, and biological systems. Sufficient detail is given to provide an overview of each topic, and there are short reference lists at the end of each chapter for the reader who wants to dive more deeply into a particular topic. A variety of applications are presented throughout the text, and the book culminates with a chapter on "Nanomachines and Nanodevices." This is an excellent way to complete the introduction to nanotechnology, as it highlights systems and applications that integrate the nanoscale systems discussed in the previous chapters.

There are no exercises, so this is not an ideal textbook, but it is a very nice reference text and gives a solid overview of topics and techniques that could be included in an introductory class on nanotechnology. For a course, this book provides basics that instructors could develop more fully as they see fit.

Reviewer: Linda Olafsen is an assistant professor in the Department of Physics and Astronomy at the University of Kansas and is a member of the MRS Bulletin Book Review Board. Her semiconductor physics research is focused on the study of optical and electronic properties of antimonide-based heterostructures for the development of more efficient, high-temperature semiconductor lasers and detectors in the near-and mid-infrared.

\section{Handbook of Superconducting Materials}

David A. Cardwell and David S. Ginley, Eds.

(Institute of Physics, Bristol, U.K., 2002)

2122 pages; $\$ 610.00$

ISBN: 0-7503-0898-2

I had been reluctant to open the book for a while after I received it, simply because it contains two thick, heavy volumes. After taking a careful look at this handbook, I was then attracted and later overwhelmed by the thoroughness of the book in its attempt to cover the physics, processing, materials, characterization, and applications of superconductors, both the low $-T_{c}$ metals/alloys and high- $T_{c}$ cuprates.

Superconductivity research has been an active area in condensed-matter physics research since Onnes discovered the first superconductor in 1911. The discovery of the high- $T_{c}$ cuprates by Bednorz and Müller in 1986 generated a renewed interest in superconductivity that has grown into a huge subfield involving thousands of researchers worldwide. Although many of the research results in superconductivity have been published as highly technical articles over the years, it is difficult for a graduate student or someone new in the field to grasp the "basics" of superconductivity. In addition, even for an experienced researcher, it remains challenging to reformulate state-of-the-art knowledge in a systematic way.

This handbook of superconducting materials succeeds in achieving this and provides an excellent resource on the basic knowledge of superconductivity to a beginner in the field. One can easily find answers to common questions like, What is superconductivity? What superconductors have been discovered so far? What are the methods developed for synthesis and epitaxy of bulk and thin-film superconductors? What are the applications of superconductivity? An experienced researcher would also find this book extremely interesting and helpful with a large number of highly correlated review articles written in tutorial styles. In fact, this is not surprising, considering that more than 120 world experts in superconductivity were involved in writing this book. I have no doubt that this book will become a bench-top reference in many laboratories.

The book could be more useful if its digital version is made available in the near future. Besides the convenience of printing out a specific article in the book, the digital version may greatly facilitate searches for specific information.

Reviewer: Judy $W u$ is a professor in the Department of Physics and Astronomy at the University of Kansas. Her research topics include thin films, high- $\mathrm{T}_{c}$ superconducting films, boron nanowires, and multichannel scanning probe microscopy.

\section{Innovative Processing and Synthesis of Ceramics, Glasses, and Composites VII: Ceramic Transactions Vol. 154}

J.P. Singh and N.P. Bansal, Eds.

(The American Ceramic Society,

Westerville, $\mathrm{OH}, 2003)$

335 pages, $\$ 109.00$

ISBN 1-57498-208-7

Advanced ceramics, glasses, and composites are employed in a wide variety of structural and functional applications. As a consequence, the continued development of improved processing methods for these materials is the subject of significant research effort around the world, with much of this work reported through the continuing series of Ceramic Transactions conference proceedings published by the American Ceramic Society. With 29 contributed papers, the present volume summarizes recent progress in this area of advanced materials processing.

There is a relatively even emphasis on materials for both functional and structural applications, with the volume divided uniformly among papers on powder preparation, polymer precursors and solgel processing, colloidal forming methods, and thick- and thin-film development. It is notable that there is minimal coverage of densification technologies, as this is an 
extremely important aspect of processing. With the general focus on component manufacture in the "green" state, it is also perhaps a little surprising that there is just a single article on applying rapid prototyping to ceramic processing, an area that has received significant attention in recent years. Similarly, nanostructured materials are largely ignored, with the exception of three papers on various aspects of carbide powder synthesis and one on the preparation of nanostructured copper oxide powders. Given the current high level of interest in the development and characterization of nanostructured ceramics and composites, and the difficulties that are typically encountered during their preparation, this is a little disappointing. Several of the contributed articles expand on the basic premise of processing to describe the important interrelationships among processing, microstructure, and properties; this approach is desirable from the perspective of gaining a more complete understanding of the factors controlling these interrelationships. Overall, the strengths of this volume lie specifically in the areas of powder, fiber, and thick- and thin-film preparation, with several techniques reported for the synthesis of each.

As with the majority of American Ceramic Society proceedings volumes, the technical quality of the contributed articles is high, with a peer-review process invariably being applied; it should be noted that several of these articles would be acceptable for journal publication. However, the quality of figure reproduction could be improved for several of the articles. In particular, some of the micrographs have extreme contrast, essentially being black and white, while a few graphs are printed too small to make them clearly legible. Similarly, one contributed paper is entirely in bold text. As the articles are printed camera-ready, these issues should really be picked up during the peer-review process.

This volume will likely be of interest to anyone involved in the processing and development of advanced ceramics, glasses, and inorganic composites. While it does not contain any papers offering dramatic step-changes in processing technology or understanding, it does highlight the current state of the art in a number of evolving processing areas.

Reviewer: Kevin Plucknett is an associate professor in the Department of Mining and Metallurgical Engineering at Dalhousie University, Halifax, Nova Scotia, Canada. His research interests are in the area of processing-microstructure-property relationships of advanced ceramics and their composites.

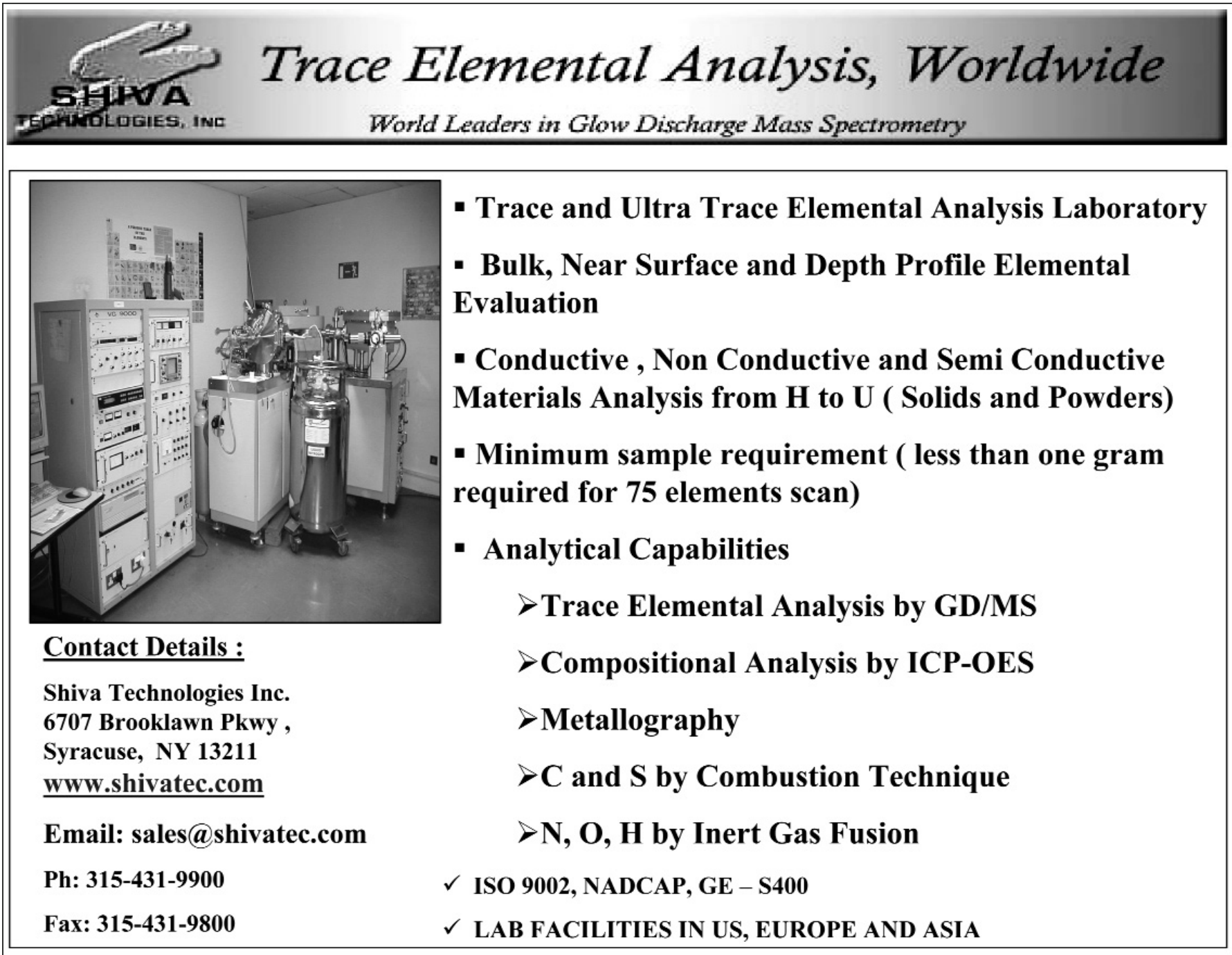

For more information, see http://advertisers.mrs.org 